\title{
Some Physiological Responses of Sheep Fed Bagasse Treated With Different Chemical Reagents
}

\author{
A.K.I. Abd-Elmoty ${ }^{(1)}$, G.A. Hassan ${ }^{(2)}$, S.T.M. Fahmy ${ }^{(1)}$ and \\ S.A.M. Sayed ${ }^{(1)}$ \\ (1) Fac. Agric., Minia Univ., and (2) Fac. Agric., Alexandria Univ., \\ Egypt.
}

\begin{abstract}
T HE Changes in some physiological parameters of sheep fed sugar cane bagasse treated with alkali and acid to improve its nutritive value were investigated. In summer $4 \mathrm{Ossimi}$ rams were fed untreated bagasse, $5 \% \mathrm{NaOH}, 2.5 \% \mathrm{NaOH}+2.5 \% \mathrm{Ca}(\mathrm{oH})_{2}$ and $6 \% \mathrm{NH}_{3}$ treated bagasse in a $4 \times 4$ latin aquare arrangement. In winter this experiment was repeated with an addition of $2.4 \% \mathrm{H}_{2} \mathrm{SO}_{4}$ treatment in a $5 \times 5$ latin square arrangement. Chemical treatment of bagasse had insignificant effect on the physiological responses (rectal temperature, pulse rate and respiration rate), on blood hemoglobin and white blood cell counts, on serum total protein, albumin globulin and glucose, on plasma osmolality, sodium and potassium values. Values of packed cell volume and red blood counts decreased $(\mathrm{P}<0.01)$ by $\mathrm{NaOH}$ treatment. Urine osmolality, sodium and potassium values were higher $(\mathrm{P}<0.01)$ in both $\mathrm{NaOH}$ and $\mathrm{NaOH}+\mathrm{Ca}(\mathrm{OH})_{2}$ treatments than control. Summer season induced significant $(\mathrm{P}<0.01)$ decreases in blood hemoglobin and packed cell volume and in urine sodium and potassium, and significant increases in rectal temperature, respiration rate $(P<0.01)$ white blood cell counts $(P<0.05)$ and in serum total protein and albumin $(\mathrm{P}<0.01)$.
\end{abstract}

Key words : Sheep, Physiological response, bagasse feeding

Sugar cane bagasse can be used as alternative feed for ruminants to partially fill the gap in available feeds. In general, the feeding value of bagasse, as a roughage, can be improved through physical, microbiological or chemical treatments. The improvement of feeding value of roughages achieved through the chemical treatments depends on several factors e.g., the treated stuff, the chemical reagents used, reagent concentration, ... etc. (Sundstol and Coxworth, 1984). Feeding on chemically treated roughages induce marked changes in several physiological parameters in animals fed the treated ration (Salem, 1980; Klimer et al. 1981; Vincent et al., 1987). The aim of this study was to determine the changes in the physiological response of sheep fed on sugar cane bagasse treated with different alkali or acid detergents. 


\section{Material and Methods}

The present study was carried out at the Experimental Farm, Faculty of Agriculture, Minia University. The animals used were Ossimi rams at $10-12$ months of age and 30 - $40 \mathrm{~kg}$ body weight. All animals were individually confined in digestion crates which were maintained in semi-open stable.

During summer 4 rams were fed on 4 different chemically treated bagasse in a $4 \times 4$ latin squar design. The treatments were as follows : untreaed sugar cane bagasse (control), sugar cane bagasse treated with $5 \% \mathrm{NaOH}$, sugar cane bagasse treated with $2.5 \% \mathrm{NaOH}+2.5 \% \mathrm{Ca}(\mathrm{OH})_{2}$ and sugar cane bagasse treated with $6 \% \mathrm{NH} 4 \mathrm{OH}$. One more treatment (Sugar cane bagasse treated with $2.4 \% \mathrm{H}_{2} \mathrm{SO}_{4}$ ) was added during the winter experiment, and a $5 \times 5$ latin squar design was used. The bagasse represented $75 \%$ of the diet, while the rest $(25 \%)$ was a concentrate mixture. Animals were fed ad-libitum on experimental diets for a period of 17 days. After 10 days of adaptation on the new feed, physiological parameters were recorded daily for a period of 7 days. Rectal temperature, pulse rate and respiration rate of all animals were measurud twice a day at $8.30 \mathrm{a} . \mathrm{m}$ and 4.00 p.m. Three blood samples were obtained from the jugular vien of each animal. Blood collection was carried out every other day during the 7 day period. In part of blood sample EDTAwas used as anticoagulant. Plasma or serum was obtained by centrifugation of blood at 3000 r.p.m. for 20 minutes, and were stored at $20 \mathrm{C}$ until used for analysis. Whole blood samples were analyzed for hemoglobin $(\mathrm{Hb})$ concentration (Eilers, 1967) red blood cell (RBC) and white blood cell (WBC) counts (using Bright line hemocytometer) and packed cell volum (using microhematocrit tubes and hematocrit centrifuge). Total serum protein (TP), albumin (A) and glucose were determined using commercial kits (Bio-Merieux, France). Globulin (G) concentration was obtained by substracting the values of albumin from the corresponding values of total protein. Sodium $(\mathrm{Na})$ and potassium $(\mathrm{K})$ concentration were measured in plasma and urine samples with flame photometer. Plasma and urine osmolality were measured using osmometer (osmett A 5002). The average air temperature, relative humidity and vapour pressure were $22.2^{\circ} \mathrm{C}, 64.2 \%$ and 7.4 mbar during summer and were $11.0^{\circ} \mathrm{C}$, $68.4 \%$ and 3.1 mbar during winter. Data obtained were stbjected to statistical analysis according to Snedecor and Cochran (1980).

\section{Results and Discussion}

1- Rectal temperature (RT) respiration rate $(R R)$ and pulse rate (PR)

Data presented in Table 1 show that chemical treatments of bagasse casued no significant effect on these parameters either in summer or in winter. The overall mean of $R T, R R$ and PR were higher during afternoon than at morning. Also, the mean values of $R T$ and $R R$ were significiantly higher $(P<0.01)$ during summer than during winter, while in PR was not significantly affected by season. There is a relationship

Egypt.J.Anim.Prod., 28, No.2 (1991) 
TABLE 1. Means ( \pm SE) of rectal temperature $(R T)$, respiration rate $(R R)$ and pulse rate (PR) in Ossimi rams fed sugar cane bagasse treated with different chemicals in summer and winter seasons.

\begin{tabular}{|c|c|c|c|c|c|c|c|c|c|}
\hline \multirow[t]{2}{*}{ Treatment } & \multicolumn{3}{|c|}{ RT (C-) } & \multicolumn{3}{|c|}{ RR (res. $/ \mathrm{min}$.) } & \multicolumn{3}{|c|}{ PR (pulse / min.) } \\
\hline & a.m. & p.m. & Average & a.m. & p.m. & Average & a.m. & p.m. & Average \\
\hline \multirow{2}{*}{$\begin{array}{l}\text { Summer } \\
\text { Untreated }\end{array}$} & & & N.S & & & N.S & & & N.S \\
\hline & 38.8 & 39.1 & $38.9 \mathrm{a}$ & 23.5 & 30.6 & $27.1 \mathrm{a}$ & 51.0 & 54.8 & $52.9 \mathrm{a}$ \\
\hline \multirow[b]{2}{*}{$\mathrm{NaOH}$} & \pm 0.25 & \pm 0.20 & \pm 0.14 & \pm 3.59 & \pm 6.02 & \pm 4.77 & \pm 9.31 & \pm 8.94 & \pm 9.08 \\
\hline & 38.9 & 39.2 & 39.1 a & 24.5 & 30.7 & $27.6 \mathrm{a}$ & 63.9 & 70.7 & $67.3 \mathrm{a}$ \\
\hline \multirow{3}{*}{$\mathrm{NaOH}+\mathrm{Ca}(\mathrm{OH})_{2}$} & \pm 0.15 & \pm 0.13 & \pm 0.14 & \pm 1.69 & \pm 2.77 & \pm 2.13 & \pm 8.36 & \pm 10.48 & \pm 9.37 \\
\hline & 239.1 & 39.4 & $39.2 \mathrm{a}$ & 31.2 & 39.2 & $35.2 \mathrm{a}$ & 62.8 & 66.5 & $64.6 \mathrm{a}$ \\
\hline & \pm 0.14 & \pm 0.17 & \pm 0.15 & \pm 6.28 & \pm 8.97 & \pm 7.62 & \pm 9.53 & \pm 10.19 & \pm 9.84 \\
\hline \multirow{2}{*}{$\mathrm{NH}_{4} \mathrm{OH}$} & 39.9 & 39.3 & $39.1 \mathrm{a}$ & 30.3 & 39.9 & $35.0 \mathrm{a}$ & 57.1 & 62.9 & $60.0 \mathrm{a}$ \\
\hline & \pm 0.26 & \pm 0.14 & \pm 0.20 & \pm 7.56 & \pm 8.06 & \pm 7.70 & \pm 5.48 & \pm 5.87 & \pm 5.64 \\
\hline \multicolumn{3}{|l|}{ Winter } & N.S & & & N.S & & & N.S \\
\hline \multirow{2}{*}{ Untreated } & 38.1 & 39.1 & $38.6 \mathrm{a}$ & 12.4 & 14.1 & $13.3 \mathrm{a}$ & 58.0 & 64.1 & $61.0 \mathrm{a}$ \\
\hline & \pm 8.34 & \pm 0.10 & \pm 0.20 & \pm 1.73 & \pm 1.93 & \pm 1.83 & \pm 4.50 & \pm 5.57 & \pm 4.97 \\
\hline \multirow{2}{*}{$\mathrm{NaOH}$} & 38.1 & 39.0 & $38.6 \mathrm{a}$ & 13.4 & 15,4 & $14.6 \mathrm{a}$ & 62.2 & 67.2 & $64.7 \mathrm{a}$ \\
\hline & \pm 0.24 & \pm 0.12 & \pm 0.13 & \pm 1.39 & \pm 1.65 & \pm 1.43 & \pm 3.53 & \pm 4.16 & \pm 3.83 \\
\hline \multirow{2}{*}{$\mathrm{NaOH}+\mathrm{Ca}(\mathrm{OH})_{2}$} & 238.4 & 39.3 & $38.9 \mathrm{a}$ & 12.6 & 15.0 & $13.8 \mathrm{a}$ & 65.2 & 69.6 & $67.4 \mathrm{a}$ \\
\hline & \pm 0.37 & \pm 0.22 & \pm 0.28 & \pm 0.99 & \pm 1.70 & \pm 1.34 & \pm 3.41 & \pm 5.02 & \pm 4.18 \\
\hline \multirow{2}{*}{$\mathrm{NH} 4 \mathrm{OH}$} & 38.1 & 39.1 & $38.6 \mathrm{a}$ & 13.8 & 17.4 & $15.6 \mathrm{a}$ & 59.3 & 64.8 & $62.0 \mathrm{a}$ \\
\hline & \pm 0.22 & \pm 0.14 & \pm 0.14 & \pm 1.49 & \pm 2.94 & \pm 2.19 & \pm 2.07 & \pm 1.83 & \pm 1.91 \\
\hline \multirow{2}{*}{$\mathrm{H}_{2} \mathrm{SO}_{4}$} & 38.1 & 39.1 & $38.5 \mathrm{a}$ & 12.0 & 14.4 & $13.2 \mathrm{a}$ & 55.2 & 58.6 & $59.9 \mathrm{a}$ \\
\hline & \pm 0.17 & \pm 0.12 & \pm 0.12 & \pm 0.87 & \pm 1.38 & \pm 1.12 & \pm 7.20 & \pm 7.26 & \pm 7.22 \\
\hline Untreated & & & N.S & & & N.S & & & N.S \\
\hline \multirow{3}{*}{$\mathrm{NaOH}$} & & & & & & & & & \\
\hline & 38.4 & 39.1 & $38.7 \mathrm{a}$ & 17.4 & 21.4 & $19.4 \mathrm{a}$ & 45.9 & 60.0 & $57.4 \mathrm{a}$ \\
\hline & \pm 0.24 & \pm 0.10 & \pm 0.13 & +2.60 & \pm 3.93 & \pm 3.25 & \pm 4.64 & \pm 4.69 & \pm 4.76 \\
\hline \multirow{2}{*}{$\mathrm{NaOH}+\mathrm{Ca}(\mathrm{OH})_{2}$} & 238.4 & 39.0 & $38.8 \mathrm{a}$ & 18.3 & 22.2 & $20.4 \mathrm{a}$ & 59.6 & 68.7 & $65.8 \mathrm{a}$ \\
\hline & \pm 0.20 & \pm 0.12 & \pm 0.12 & \pm 2.20 & \pm 3.04 & \pm 2.57 & \pm 5.91 & \pm 4.85 & \pm 4.35 \\
\hline \multirow{2}{*}{$\mathrm{NH}_{4} \mathrm{OH}$} & 38.7 & 39.3 & $39.0 \mathrm{a}$ & 20.9 & 25.8 & $23.3 \mathrm{a}$ & 64.1 & 68.2 & $66.1 \mathrm{a}$ \\
\hline & \pm 0.23 & \pm 0.13 & \pm 0.17 & \pm 4.18 & \pm 5.68 & \pm 4.93 & \pm 4.31 & \pm 4.96 & \pm 4.61 \\
\hline \multirow{2}{*}{$\mathrm{H}_{2} \mathrm{SO}_{4}$} & 38.5 & 39.2 & $38.8 \mathrm{a}$ & 21.1 & 27.4 & $24.2 \mathrm{a}$ & 58.4 & 63.9 & $61.1 \mathrm{a}$ \\
\hline & \pm 0.21 & \pm 0.10 & \pm 0.14 & \pm 4.30 & \pm 5.36 & \pm 4.78 & \pm 2.52 & \pm 2.61 & \pm 2.54 \\
\hline Season & & & *** & & & "** & & & N.S \\
\hline \multirow{2}{*}{ Summer } & & & & & & & & & \\
\hline & 38.9 & 39.2 & $39.1 \mathrm{a}$ & 27.4 & 35.1 & $31.2 \mathrm{a}$ & 58.7 & 63.7 & $61.2 \mathrm{a}$ \\
\hline \multirow{3}{*}{ Winter } & \pm 0.09 & \pm 0.07 & \pm 0.08 & \pm 2.52 & \pm 3.28 & \pm 2.87 & \pm 3.95 & \pm 4.32 & \pm 4.12 \\
\hline & 38.2 & 39.1 & $38.7 \mathrm{~b}$ & 13.1 & 15.5 & $14.3 \mathrm{~b}$ & 61.1 & 66.4 & $63.8 \mathrm{a}$ \\
\hline & \pm 0.14 & \pm 0.07 & \pm 0.09 & \pm 0.66 & \pm 1.01 & \pm 0.82 & \pm 1.73 & \pm 2.07 & \pm 1.87 \\
\hline
\end{tabular}


between rising air temperaure during summer or during afternoon and the increase in the values of RT and RR. RT and RR have a satisfactory mean to detect the response of widely used by several investigators as Khalil (1980); Taher (1985) and El-Nage of animals to environmental temperature. RR in sheep during summer, while minimur (1987) noted maximum values of RT and higher RT and RR values were recornim values were noted during winter. Also, early morning (Younis, et al, 1977; Taher, 1985). afternoon than those recorded in were studied by several investigators (Shal, 1985). Diurnal variations in PR in sheep in the afternoon than in the morning.

2- Hemoglobin (Hb), red blood cell (RBC) counts, white blood cell (WBC) counts and
packed cell volume (PCV)

Data presented in Table 2 show that chemical treatments of bagasse caused no counts during summer and WBC counts either in summer or in winter, while RBC treatment. During winter $\mathrm{NCV}$ values during winter were significantly affected by significant decline $(\mathrm{P}<0.01)$ (control). Treatment with $\mathrm{H}_{2} \mathrm{SO}$ a $\mathrm{RBC}$ counts also decreased $\mathrm{P}_{4}$ also reduced $\mathrm{PCV}$, but the effect was not significant. summer, but treatment with $\mathrm{NaOH}+\mathrm{Ca}(\mathrm{OH}) 2$ during the basse with $\mathrm{NaOH}$ during $(\mathrm{P}<0.01)$ in $\mathrm{RBC}$ counts.

Generally, in overall means there was a tendency for a decrease in $\mathrm{Hb}, \mathrm{RbC}, \mathrm{WBC}$ and PCV values when animals were fed the treated bagasse. NaOH treatment had the greatest effect on these hematological characteristics than other treatments. These data maize stalks in agreement with results of Salem (1980) who found that treatments of values in sheep when straw with $\mathrm{NaOH}$ and $\mathrm{Ca}(\mathrm{OH})_{2}$ decreased $\mathrm{Hb}, \mathrm{RBC}$ and $\mathrm{WBC}$ reported that treatment compared with untreated roughages. Also, Klimer et al. (1981) $\mathrm{RBC}$ and PCV and C

There was significant effect of season on blood $\mathrm{Hb}$, WBC counts and PCV values. The mean values of $\mathrm{Hb}$ and $\mathrm{PCV}$ were lower $(\mathrm{P}<0.01)$ in summer than in winter, while that high environmental $(\mathrm{P}<0.05)$ in summer than in winter. Studies on cows also indicated leucocyte counts and a temperature during summer caused an elevation in the decline in blood $\mathrm{Hb}$ and PCV tissue fluid is transfered the hemodilution of blood, as more from the body surface. Lee et al season, which was partially attributed to a reported a decline in PCV during the hot increased rate of $\mathrm{RBC}$ destruction.

Egypt. J.Anim. Prod., 28, No.2 (1991) 
TABLE 2. Means ( \pm SE) of hemoglobin (Hb) concentration, red blood cell (RBC) counts, packed cell volume (PCV) and white blood cell (WBC) counts in ossimi rams fed sugar cane bagasse treated with different chemicals in summer and winter seasons.

\begin{tabular}{|c|c|c|c|c|}
\hline Treatment & $\begin{array}{c}\mathrm{Hb} \\
(\mathrm{gm} / 100 \mathrm{ml})\end{array}$ & $\begin{array}{c}\text { RBC } \\
\left(X 10^{6} / \mathrm{ml}\right)\end{array}$ & $\begin{array}{c}\text { WBC } \\
\left(\mathrm{X} 10^{3} / \mathrm{ml}\right)\end{array}$ & $\begin{array}{l}\text { PCV } \\
(\%)\end{array}$ \\
\hline $\begin{array}{l}\text { Summer } \\
\text { Untreated } \\
\mathrm{NaOH} \\
\mathrm{NaOH}+\mathrm{Ca}\left(\mathrm{OH}_{2}\right. \\
\mathrm{H}_{4} \mathrm{OH}\end{array}$ & $\begin{array}{c}\text { N.S } \\
13.1 \pm 0.21 \mathrm{a} \\
11.5 \pm 0.47 \mathrm{a} \\
12.6 \pm 0.40 \mathrm{a} \\
12.8 \pm 0.51 \mathrm{a}\end{array}$ & $\begin{array}{c}* * \\
7.5 \pm 0.44 \mathrm{a} \\
6.2 \pm 0.21 \mathrm{~b} \\
8.3 \pm 0.44 \mathrm{c} \\
7.5+0.27 \mathrm{a}\end{array}$ & $\begin{array}{c}\text { N.S } \\
9.6 \pm 2.16 \mathrm{a} \\
7.6 \pm 1.17 \mathrm{a} \\
10.1 \pm 0.99 \mathrm{a} \\
7.3 \pm 0.75 \mathrm{a}\end{array}$ & $\begin{array}{c}\text { N.S } \\
30.6 \pm 0.98 \text { a } \\
28.4 \pm 1.12 \text { a } \\
29.4 \pm 2.26 \text { a } \\
32.5 \pm 2.28 \text { a }\end{array}$ \\
\hline $\begin{array}{l}\text { Winter } \\
\text { Untreated } \\
\mathrm{NaOH} \\
\mathrm{NaOH}+\mathrm{Ca}(\mathrm{OH})_{2} \\
\mathrm{NH}_{4} \mathrm{OH} \\
\mathrm{H}_{2} \mathrm{SO}_{4}\end{array}$ & $\begin{array}{c}\text { N.S } \\
14.5 \pm 0.57 \mathrm{a} \\
14.3 \pm 0.70 \mathrm{a} \\
14.3 \pm 0.44 \mathrm{a} \\
14.2 \pm 0.25 \mathrm{a} \\
14.7 \pm 0.60 \mathrm{a}\end{array}$ & $\begin{array}{c}\text { N.S } \\
7.6 \pm 0.23 \mathrm{a} \\
6.8 \pm 0.61 \mathrm{a} \\
7.4 \pm 0.31 \mathrm{a} \\
7.7 \pm 0.28 \mathrm{a} \\
7.8 \pm 0.43 \mathrm{a}\end{array}$ & $\begin{array}{c}\text { N.S } \\
7.9 \pm 0.56 \mathrm{a} \\
6.8 \pm 0.24 \mathrm{a} \\
7.0 \pm 0.22 \mathrm{a} \\
7.6 \pm 0.18 \mathrm{a} \\
6.9 \pm 0.47 \mathrm{a}\end{array}$ & $\begin{array}{c}{ }^{* *} \\
39.0 \pm 1.17 \mathrm{a} \\
33.2 \pm 0.44 \mathrm{~b} \\
35.3 \pm 2.05 \mathrm{c} \\
35.4 \pm .0 .82 \mathrm{c} \\
36.7 \pm 1.42 \mathrm{a}\end{array}$ \\
\hline $\begin{array}{l}\text { Overall means } \\
\text { Untreated } \\
\mathrm{NaOH} \\
\mathrm{NaOH}+\mathrm{Ca}(\mathrm{OH})_{2} \\
\mathrm{NH} 4 \mathrm{OH}\end{array}$ & $\begin{array}{c}\text { N.S } \\
13.9 \pm 0.41 \mathrm{a} \\
13.0 \pm 0.64 \mathrm{a} \\
13.0 \pm 0.31 \mathrm{a} \\
13.6 \pm 0.34 \mathrm{a}\end{array}$ & $\begin{array}{c}* * \\
7.7 \pm 0.26 \mathrm{a} \\
6.6 \pm 0.18 \mathrm{~b} \\
8.2 \pm 0.41 \mathrm{c} \\
7.6 \pm 0.19 \mathrm{a}\end{array}$ & $\begin{array}{c}\text { N.S } \\
8.7 \pm 0.98 \mathrm{a} \\
7.1 \pm 0.51 \mathrm{a} \\
8.4 \pm 0.69 \mathrm{a} \\
7.4+0.33 \mathrm{a}\end{array}$ & $\begin{array}{c}* * \\
35.3 \pm 1.64 \mathrm{a} \\
31.0 \pm 0.98 \mathrm{~b} \\
32.7 \pm 1.76 \mathrm{a} \\
34.1 \pm 1.15 \mathrm{a}\end{array}$ \\
\hline $\begin{array}{l}\text { Season } \\
\text { Summer } \\
\text { Winter }\end{array}$ & $\begin{array}{c}* * * \\
12.5 \pm 0.24 \mathrm{a} \\
14.1 \pm 0.26 \mathrm{~b}\end{array}$ & $\begin{array}{c}\text { N.S } \\
7.6 \pm 0.32 \mathrm{a} \\
7.5 \pm 0.21 \mathrm{a}\end{array}$ & $\begin{array}{c}* \\
8.7 \pm 0.70 \mathrm{a} \\
7.3 \pm 0.19 \mathrm{~b}\end{array}$ & $\begin{array}{l}* * \\
30.2+089 \text { a } \\
35.7 \pm 0.75 \text { b }\end{array}$ \\
\hline
\end{tabular}

Within columns, treatment means with different letters differ significantly $\left(^{*}: \mathrm{P}<0.05 ;{ }^{*} * \mathrm{P}<\right.$ 0.01 , N S : not significant).

\section{3- Serum total protein, albumin, globulin and glucose}

Chemical treatments of bagasse had no significant effect on serum total protein, albumin and globulin concentrations and albumin/globulin ratio either in summer or in winter (Table 3). However, slightly lower total protcin and globulin values were recorded in animals fed on alkali-treated bagasse during winter. Acid treatment caused a slight increase in total prortein and globulin concentration during winter. Similar results were reported by Klimer et al (1981) who found that serum total protein, albumin and globulin concentrations in cows were not significantly affected by $0.8 \%$ $\mathrm{NaOH} \mathrm{CO}_{3}$ treated com silage. 
TABLE 3. Means ( \pm SE) of serum total proteins, albumin (A), globulin (G), A/G ratio and serum glucose in Ossimi rams fed sugar cane bagasse treated with different chemicals in summer and winter seasons.

\begin{tabular}{|c|c|c|c|c|c|}
\hline Treatment & $\begin{array}{l}\text { Total proteins } \\
(\mathrm{gm} / 100 \mathrm{ml})\end{array}$ & $\begin{array}{l}\text { Albumin (A) } \\
(\mathrm{gm} / 100 \mathrm{ml})\end{array}$ & $\begin{array}{l}\text { Globulin(G) } \\
(\mathrm{gm} / 100 \mathrm{ml})\end{array}$ & $\begin{array}{l}\mathrm{A} / \mathrm{G} \\
\text { ratio }\end{array}$ & $\begin{array}{c}\text { Glucose } \\
(\mathrm{mg} / 100 \mathrm{ml})\end{array}$ \\
\hline Summer & N.S & N.S & N.S & N.S & N.S \\
\hline Untreated & $8.0 \pm 0.33 \mathrm{a}$ & $4.1 \pm 0.06 \mathrm{a}$ & $3.9 \pm 0.27 \mathrm{a}$ & $1.1 \pm 0.06 \mathrm{a}$ & $47.1 \pm 2.18 \mathrm{a}$ \\
\hline $\mathrm{NaOH}$ & $8.0 \pm 0.40 \mathrm{a}$ & $3.8 \pm 0.24 \mathrm{a}$ & $4.1 \pm 0.35 \mathrm{a}$ & $1.0 \pm 0.11 \mathrm{a}$ & $47.5 \pm 1.00 \mathrm{a}$ \\
\hline $\mathrm{NaOH}+\mathrm{Ca}(\mathrm{OH})_{2}$ & $7.9 \pm 0.09 \mathrm{a}$ & $3.9 \pm 0.11 \mathrm{a}$ & $4.0 \pm 0.13 \mathrm{a}$ & $1.0 \pm 0.05 \mathrm{a}$ & $50.0 \pm 1.40 \mathrm{a}$ \\
\hline $\mathrm{NH}_{4} \mathrm{OH}$ & $8.1 \pm 0.16 \mathrm{a}$ & $4.0 \pm 0.17 \mathrm{a}$ & $4.1 \pm 0.08 \mathrm{a}$ & $1.0 \pm 0.05 \mathrm{a}$ & $50.4 \pm 0.70 \mathrm{a}$ \\
\hline Winter & N.S & N.S & N.S & N.S & N.S \\
\hline Untreated & $7.0 \pm 0.25 \mathrm{a}$ & $3.2 \pm 0.28 \mathrm{a}$ & $3.9 \pm 0.52 \mathrm{a}$ & $0.9 \pm 0.21$ a & $49.0 \pm 1.90 \mathrm{a}$ \\
\hline $\mathrm{NaOH}$ & $6.9 \pm 0.20 \mathrm{a}$ & $3.3 \pm 0.19 \mathrm{a}$ & $3.6 \pm 0.27$ a & $1.0 \pm 0.12 \mathrm{a}$ & $48.4 \pm 1.30 \mathrm{a}$ \\
\hline $\mathrm{NaOH}+\mathrm{Ca}(\mathrm{OH})_{2}$ & $6.9 \pm 0.28 \mathrm{a}$ & $3.1 \pm 0.15 \mathrm{a}$ & $3.8 \pm 0.39 \mathrm{a}$ & $0.9 \pm 0.13 \mathrm{a}$ & $50.4 \pm 1.50 \mathrm{a}$ \\
\hline $\mathrm{NH}_{4} \mathrm{OH}$ & $6.7 \pm 0.15 \mathrm{a}$ & $3.1 \pm 0.15 \mathrm{a}$ & $3.6+0.26 \mathrm{a}$ & $0.9 \pm 0.12 \mathrm{a}$ & $50.0 \pm 1.60 \mathrm{a}$ \\
\hline $\mathrm{H}_{2} \mathrm{SO}_{4}$ & $7.2 \pm 0.20 \mathrm{a}$ & $3.1+0.14 \mathrm{a}$ & $4.0 \pm 0.21 \mathrm{a}$ & $0.8 \pm 0.09 \mathrm{a}$ & $52.0 \pm 2.40 \mathrm{a}$ \\
\hline Qverall means & N.S & N.S & N.S & N.S & N.S \\
\hline Untreated & $7.5 \pm 0.25 \mathrm{a}$ & $3.6 \pm 0.22 \mathrm{a}$ & $3.9 \pm 0.29$ a & $1.0 \pm 0.12 \mathrm{a}$ & $48.2 \pm 1.38 \mathrm{a}$ \\
\hline $\mathrm{NaOH}$ & $7.4 \pm 0.27 \mathrm{a}$ & $3.5 \pm 0.17 \mathrm{a}$ & $3.8 \pm 0.22 \mathrm{a}$ & $1.0 \pm 0.07 \mathrm{a}$ & $48.0 \pm 0.81 \mathrm{a}$ \\
\hline $\mathrm{NaOH} \pm \mathrm{Ca}(\mathrm{OH})_{2}$ & $7.3 \pm 0.23 \mathrm{a}$ & $3.5 \pm 0.17 \mathrm{a}$ & $3.9 \pm 0.22 \mathrm{a}$ & $0.9 \pm 0.07 \mathrm{a}$ & $50.2 \pm 0.96 \mathrm{a}$ \\
\hline $\mathrm{NH}_{4} \mathrm{OH}$ & $7.3 \pm 0.27 \mathrm{a}$ & $3.5 \pm 0.19 \mathrm{a}$ & $3.8 \pm 0.17 \mathrm{a}$ & $0.9 \pm 0.07 \mathrm{a}$ & $50.2 \pm 1.10 \mathrm{a}$ \\
\hline Season & ** & $\bullet$ & N.S & N.S & N.S \\
\hline Summer & $8.0 \pm 0.12 \mathrm{a}$ & $4.0 \pm 0.08 \mathrm{a}$ & $4.0 \pm 0.11 \mathrm{a}$ & $1.0 \pm 0.03 \mathrm{a}$ & $48.8 \pm 0.74 \mathrm{a}$ \\
\hline Winter & $6.9 \pm 0.11 \mathrm{~b}$ & $3.2 \pm 0.09 \mathrm{~b}$ & $3.7 \pm 0.18 \mathrm{a}$ & $0.9 \pm 0.07 \mathrm{a}$ & $50.5 \pm 0.88 \mathrm{a}$ \\
\hline
\end{tabular}
Within columns, treatment means with different letiers differ significantly $(*$ : $\mathrm{P}<0.05 ; * *: \mathrm{P}<0.01$, NS :
not significant).

The overall means of serum glucose concentration were 48.2, 48.0, 50.2 and 50.2 $\mathrm{mg} / 100 \mathrm{ml}$ for control, $\mathrm{NaOH}, \mathrm{NaOH}+\mathrm{Ca}(\mathrm{OH})_{2}$ and $\mathrm{NH}_{4} \mathrm{OH}$ treatments, respectively. From these results it can be observed that blood glucose was increased insignificantly in rams fed on bagasse treated with $\mathrm{NaOH}+\mathrm{Ca}(\mathrm{OH})_{2}$ and $\mathrm{NH}_{4} \mathrm{OH}$. Treatment with acid $\left(\mathrm{H}_{2} \mathrm{SO}_{4}\right)$ in winter had similar effect. Maglad et al. (1986) found that grounded of nut-hulls treated with $6 \% \mathrm{NaOH}$ insignificantly increased blood glucose in sheep.

Egypt. J.Anim. Prod., 28, No. 2 (1991) 
Serum total protein and albumin were significantly higher $(P<0.01)$ during summer than during winter, while globulin was slightly increased during summer. The differnce in mean values of glucose between summer and winter was not significant, but the summer value was slightly lower than that of winter. The increase in plasme total protein levels during hot seasons was reported by other warkers (Gurrini et al., 1982; More et al., 1980). They attributed the variations in total protein to the changes in albumin concentration.

\section{4- Osmolality, sodium and potassium values in plasma and urine}

Data presented in Table 4 showed no significant variations in plasma osmolality of rams fed on bagasse treated with different chemicals either in summer or in winter. However, plasma osmolality in animals fed untreated bagesse was lower than those fed alkali-treated. In urine, the control animals had significantly $(P<0.01)$ lower values of osmolality in both seasons than those of the $\mathrm{NaOH}$ and $\mathrm{NaOH}+\mathrm{Ca}(\mathrm{OH})_{2}$ treatments. $\mathrm{NH}_{4} \mathrm{OH}$ treatment during both seasons also increased urine osmolality than the control, but these increases were not significant. Season of year had no significant effect on plasma or urine osmolality.

Plasma $\mathrm{Na}$ and $\mathrm{K}$ concentreation were not significantly increased in rams fed on bagasse treated with different chemicals during summer and winter, Urinary $\mathrm{Na}$ and $\mathrm{K}$ concentration were significantly increased $(\mathrm{P}<0.01)$ by both $\mathrm{NaOH}$ and $\mathrm{NaOH}+\mathrm{Ca}$ $(\mathrm{OH})_{2}$ treated bagasse during both seasons. Acid treatment of bagasse in winter induced a significant $(\mathrm{P}<0.01)$ decline in urine $\mathrm{Na}$ and a significant $(\mathrm{P}<0.01)$ increase in urine $\mathrm{K}$ in comparison with the control diet. Season of the year had no significant effect on plasma $\mathrm{Na}$ and $\mathrm{K}$ concentration, but urine $\mathrm{Na}$ and $\mathrm{K}$ were lower $(\mathrm{P}<0.01)$ in summer than in winter. Lamm et al. (1979) found that the urinary excretion of Na was significantly higher in lambs fed wastelage treated with $2,4,8$ and $12 \% \mathrm{NaOH}$. Increased excretion of $\mathrm{Na}$ and $\mathrm{K}$ through urine would contribute to the observed increase in urine osmolality. Probably it is more useful to study the balance of these minerals and their interrelationships rather than measuring urine $\mathrm{Na}$ and $\mathrm{K}$ concentration alone.

Results of this study provided useful information about using these chemicaly treated feed, and pointed out to the possibility of using these feeds without any adverseeffects on several physiological parameters. 
TABLE 4. Means ( \pm SE) of plasma and urine osmolality and sodium and potassium concentrations in Ossiml rams fed sugar cane bagasse treated with different chemicals in summer and winter seasons.

\begin{tabular}{|c|c|c|c|c|c|c|c|c|c|}
\hline \multirow{2}{*}{ Treatment } & \multicolumn{3}{|c|}{ Plasma } & \multicolumn{6}{|c|}{ Urine } \\
\hline & $\begin{array}{l}\text { Osmolality } \\
(\mathrm{m} .0 \mathrm{smol} / \mathrm{L})\end{array}$ & $\begin{array}{l}\text { Sodium } \\
(\mathrm{m} . \mathrm{mol} / \mathrm{L})\end{array}$ & $\begin{array}{l}\text { potassium } \\
(\mathrm{m} . \mathrm{mol} / \mathrm{L})\end{array}$ & \multicolumn{2}{|c|}{$\begin{array}{c}\text { Osmolality } \\
(\mathrm{m} .0 \mathrm{mmo1} / \mathrm{L})\end{array}$} & \multicolumn{2}{|c|}{$\begin{array}{c}\text { sodium } \\
(\mathrm{m} . \mathrm{mol} / \mathrm{L})\end{array}$} & \multicolumn{2}{|c|}{$\begin{array}{l}\text { Potassium } \\
\text { (m.mol /L) }\end{array}$} \\
\hline Summer & N.S & N.S & N.S & $* *$ & & $* *$ & & ** & \\
\hline Untreatcd & $\begin{array}{l}278.0 \mathrm{a} \\
\pm 13.46\end{array}$ & $\begin{array}{l}135.7 \mathrm{a} \\
\pm 4.98\end{array}$ & $\begin{array}{l}5.2 \text { a } \\
\pm 0.43\end{array}$ & $\begin{array}{l}169.0 \\
\pm 81.10\end{array}$ & a & $\begin{array}{l}5.9 \\
\pm 0.34\end{array}$ & $\mathrm{a}$ & $\begin{array}{l}31.6 \\
\pm 3.01\end{array}$ & a \\
\hline $\mathrm{NaOH}$ & $\begin{array}{l}305.0 \mathrm{a} \\
\pm 5.63\end{array}$ & $\begin{array}{l}140.1 \text { a } \\
\pm 1.16\end{array}$ & $\begin{array}{l}5.7 \text { a } \\
\pm 1.00\end{array}$ & $\begin{array}{l}803.0 \quad b \\
\pm 185.85\end{array}$ & b & $\begin{array}{l}15.6 \\
\pm 2.63\end{array}$ & $\mathrm{~b}$ & $\begin{array}{l}75.9 \\
\pm 6.33\end{array}$ & $\mathrm{~b}$ \\
\hline $\mathrm{NaOH}+\mathrm{Ca}(\mathrm{OH})_{2}$ & $\begin{array}{l}306.0 \text { a } \\
\pm 5.41\end{array}$ & $\begin{array}{l}140.1 \text { a } \\
\pm 1.16\end{array}$ & $\begin{array}{l}6.5 \text { a } \\
\pm 0.73\end{array}$ & $\begin{array}{l}758.0 \\
\pm 170.26\end{array}$ & b & $\begin{array}{l}12.9 \\
\pm 1.60\end{array}$ & $\mathrm{~b}$ & $\begin{array}{l}73.4 \\
\pm 8.80\end{array}$ & $\mathrm{bc}$ \\
\hline $\mathrm{NH}_{4} \mathrm{OH}$ & $\begin{array}{l}303.0 \mathrm{a} \\
\pm 11.90\end{array}$ & $\begin{array}{l}137.1 \text { a } \\
\pm 2.32\end{array}$ & $\begin{array}{l}4.9 \text { a } \\
\pm 0.48\end{array}$ & $\begin{array}{l}356.0 \\
\pm 82.31\end{array}$ & a & $\begin{array}{l}5.9 \\
\pm 0.44\end{array}$ & a & $\begin{array}{l}30.1 \\
\pm 6.48\end{array}$ & a \\
\hline Winter & N.S & N.S & N.S & $* *$ & & $* *$ & & $* *$ & \\
\hline Untreated & $\begin{array}{l}318.0 \mathrm{a} \\
\pm 2.17\end{array}$ & $\begin{array}{l}135.7 \mathrm{a} \\
\pm 1.96\end{array}$ & $\begin{array}{l}5.4 \text { a } \\
\pm 0.61\end{array}$ & $\begin{array}{l}294.0 \\
\pm 49.81\end{array}$ & a & $\begin{array}{l}8.7 \\
\pm 0.95\end{array}$ & a & $\begin{array}{l}59.8 \\
\pm 8.27\end{array}$ & a \\
\hline $\mathrm{NaOH}$ & $\begin{array}{l}324.0 \mathrm{~s} \\
\pm 9.54\end{array}$ & $\begin{array}{l}141.9 \mathrm{a} \\
\pm 0.70\end{array}$ & $\begin{array}{l}5.6 \text { a } \\
\pm 0.28\end{array}$ & $\begin{array}{l}714.0 \\
\pm 90.90\end{array}$ & b & $\begin{array}{l}19.1 \\
\pm 0.99\end{array}$ & b & $\begin{array}{l}99.1 \\
\pm 5.60\end{array}$ & b c \\
\hline $\mathrm{N} 3 \mathrm{OH}+\mathrm{Ca}(\mathrm{OH})_{2}$ & $\begin{array}{l}322.0 \mathrm{a} \\
\pm 8.18\end{array}$ & $\begin{array}{l}142.4 \text { a } \\
\pm 0.81\end{array}$ & $\begin{array}{l}6.4 \text { a } \\
\pm 0.35\end{array}$ & $\begin{array}{l}678.0 \\
\pm 111.55\end{array}$ & $b c$ & $\begin{array}{l}17.8 \\
\pm 1.40\end{array}$ & $\mathrm{~b}$ & $\begin{array}{l}87.2 \\
\pm 3.52\end{array}$ & d \\
\hline $\mathrm{NH}_{4} \mathrm{OH}$ & $\begin{array}{l}323.0 \mathrm{a} \\
\pm 9.40\end{array}$ & $\begin{array}{l}141.1 \text { a } \\
\pm 3.17\end{array}$ & $\begin{array}{l}6.1 \quad a \\
\pm 0.46\end{array}$ & $\begin{array}{l}452.0 \\
\pm 56.26\end{array}$ & $\mathrm{ac}$ & $\begin{array}{l}7.6 \\
\pm 1.05\end{array}$ & $\mathrm{a}$ & $\begin{array}{l}56.3 \\
\pm 4.48\end{array}$ & a \\
\hline $\mathrm{H}_{2} \mathrm{SO}_{4}$ & $\begin{array}{l}308.0 \mathrm{a} \\
\pm 4.35\end{array}$ & $\begin{array}{l}142.2 \text { a } \\
\pm 1.59\end{array}$ & $\begin{array}{l}6.2 \quad a \\
\pm 0.40\end{array}$ & $\begin{array}{l}355.0 \\
\pm 97.10\end{array}$ & a & $\begin{array}{l}3.9 \\
\pm 0.40\end{array}$ & c & $\begin{array}{l}79.7 \\
\pm 6.69\end{array}$ & cd \\
\hline Overallmeans & N.S & N.S & N.S & $* *$ & & $* *$ & & ** & \\
\hline untreated & $\begin{array}{l}300.2 \mathrm{a} \\
\pm 8.99\end{array}$ & $\begin{array}{l}135.7 \text { a } \\
\pm 2.22\end{array}$ & $\begin{array}{l}5.3 \quad \text { a } \\
\pm 0.37\end{array}$ & $\begin{array}{l}238.4 \\
\pm 47.65\end{array}$ & a & $\begin{array}{l}7.5 \\
\pm 0.71\end{array}$ & a & $\begin{array}{l}47.2 \\
\pm 6.71\end{array}$ & a \\
\hline $\mathrm{NaOH}$ & $\begin{array}{l}315.4 \mathrm{a} \\
\pm 6.48\end{array}$ & $\begin{array}{l}141.1 \text { a } \\
\pm 0.70\end{array}$ & $\begin{array}{l}6.2 \quad a \\
\pm 0.46\end{array}$ & $\begin{array}{l}753.1 \\
\pm 91.08\end{array}$ & $b$ & $\begin{array}{l}17.5 \\
\pm 1.35\end{array}$ & $\mathrm{~b}$ & $\begin{array}{l}88.8 \\
\pm 5.65\end{array}$ & b \\
\hline $\mathrm{NaOH} \pm \mathrm{Ca}(\mathrm{OH})_{2}$ & $\begin{array}{l}315.0 \mathrm{a} \\
\pm 5.66\end{array}$ & $\begin{array}{l}141.4 \text { a } \\
\pm 0.75\end{array}$ & $\begin{array}{l}6.4 \text { a } \\
\pm 0.35\end{array}$ & $\begin{array}{l}713.7 \\
\pm 92.11\end{array}$ & b & $\begin{array}{l}15.6 \\
\pm 1.32\end{array}$ & $\mathrm{~b}$ & $\begin{array}{l}81.1 \\
\pm 4.71\end{array}$ & $\mathrm{~b}$ \\
\hline $\mathrm{NH}_{4} \mathrm{OH}$ & $\begin{array}{l}314.0 \mathrm{a} \\
\pm 7.76\end{array}$ & $\begin{array}{l}139.3 \mathrm{a} \\
\pm 2.05\end{array}$ & $\begin{array}{l}5.6 \text { a } \\
\pm 0.38\end{array}$ & $\begin{array}{l}409.3 \\
\pm 47.94\end{array}$ & a & $\begin{array}{l}6.8 \\
\pm 0.65\end{array}$ & a & $\begin{array}{l}44.7 \\
\pm 5.81\end{array}$ & $\mathbf{a}$ \\
\hline Seasen & N.S & N.S & N.S & N.S & & ** & & 4 & \\
\hline Summer & $\begin{array}{l}297.9 \mathrm{a} \\
\pm 5.29\end{array}$ & $\begin{array}{l}138.2 \mathrm{a} \\
\pm 1.38\end{array}$ & $\begin{array}{l}5.6 \quad \text { a } \\
\pm 0.35\end{array}$ & $\begin{array}{l}521.2 \\
\pm 92.87\end{array}$ & a & $\begin{array}{l}10.1 \\
\pm 1.30\end{array}$ & a & $\begin{array}{l}52.8 \\
\pm 6.36\end{array}$ & a \\
\hline Winter & $\begin{array}{l}321.8 \mathrm{a} \\
\pm 3.67\end{array}$ & $\begin{array}{l}140.3 \mathrm{a} \\
\pm 1.08\end{array}$ & $\begin{array}{l}6.1 \quad \mathrm{a} \\
\pm 0.23\end{array}$ & $\begin{array}{l}534.6 \\
\pm 5.12\end{array}$ & a & $\begin{array}{l}13.3 \\
\pm 1.30\end{array}$ & a & $\begin{array}{l}75.6 \\
\pm 4.92\end{array}$ & $\mathrm{~b}$ \\
\hline
\end{tabular}

Within columns, treatment means with different letters differ significantly $(*: \mathrm{P}<0.05 ; * *: \mathrm{P}<$ 0.01 , N.S : not significant).

Egypt. J Anim. Prod., 28, No. 2 (1991) 


\section{References}

Eilers, RJ. (1967) Notification of final adoption of an international method and standard solution for hemoglobinometry: Specific for preparation of standard solution. Am.J. Clin path. $47: 212$.

El-Naggar, M.I.Y. (1987) Changes in water requirments and metabolism in some physiological responses and hematological characteristics during lactation in sheep and goats. M.SC. Thesis, Fac. of Agric., Alex. Univ., Egypt.

EI-Nouty, F.D., Hassan, G.A. and salam, M.H (1986) Effect of season and level of * production on hematological values in Holstein cows. Indian. J. Anim. Sci. 56:346.

Gurrini, V.H., Betchinger, H. and Coster, N. (1982) The effect on sheep urinary and plasma electrolytes after 4 weeks exposure to different ambient temperatures and humidities. Aust. J. Agric. Res. $33: 347$.

Khalil, M.H. (1980) Studies on the wool coat of sheep in relation to their adaptability of the Egyptian environment. Ph.D.Thesis, Al-Azhar Univ., Cairo, Egypt.

Klimer, L.H., Muller, L.D. and Snyder, T.J. (1981) Addition of sodium bicarbonat to rations of postpartum dairy cows physiological and metabolic effects. J. Dairy Sci. $64: 357$.

Lamm, W.D., Webb, K.E. and Fontenant, J.P (1979) Ensiling characteristics, digestibility and feeding value of ensiled cattle waste and ground hay with and without sodium hydroxid. J. of Anim. Sci. $48: 1$.

Lee, J.A., Roussel, J.D. and Beatty, J.F. (1976) Effect of temperature seasons on bovine adrenal cortical function, blood profil and milk production. J. Dairy Sci. 59: 104.

Maglad, M.A., Lutfi, A.A. and Gabir, S. (1986) The effect of grinding nut hulls either with or without alkali treatments on digestibility of diet and on ruminal and blood components. Anim. Feed Sci. Technol., $15: 69$.

More, T., Ray, A.K. and Singh, M. (1980) Note on the effect of thermal exposure on body fluid composition of different breed and breed cross of sheep. Indian. J. Anim. Sci. 50 207.

Salem, O.A. I. (1980) Studies with different short-time alkali treated roughages in metabolism trial with sheep with reference to detailed nutritions, animal activity and animal behaviour. Ph.D. Thesis, Cairo Univ., Cairo.

Shalaby, T.H.A. (1985) Performance and adaptation of local sheeps to varied environmental and managerial conditions Ph. D. Thesis, Cairo Univ., Cairo. 
Snedecor, G.W. and Cochran, W.G. (1980) Statistical Methods. The Iowa State Unversity, Press. Ames. Iowa. USA.

Sundstol, F. and Coxworth, E.M. (1984) Ammonia treatment. Straw and other fibrous by product as feed. 196. 267. F. Sundstol and E. Owen (Editors) Elsevier, Netherlands.

Taher, H.H. (1985) Effect of season of the year and breed on some blood components, water requirements and metabolism in sheep and goats. M. Sc. Thesis, Fac. of Agric., Alex. Univ. Egypt.

Youns, A.A., Al-Mohmoud, F., El-Tawll, E.A. and El-Shobokshy, A.S. (1977) Performance and heat tolerance of Awassi lambs as affected by early shearing. J. Agric. Sci. Camb., $89: 565$.

Vincent, I.C., Michell, A.R., Williams, H.L. and Hill, R. (1987) Effects of sodium intake during two parities on Na status in Blackface sheep. British. J. Nut. 57: 447.

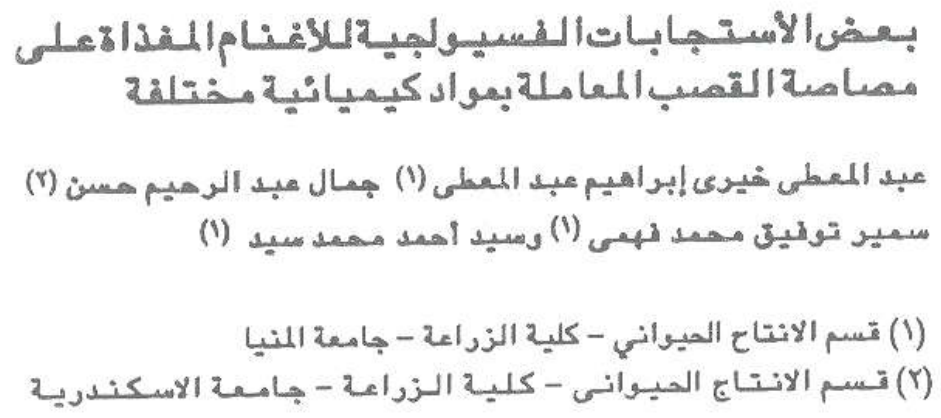

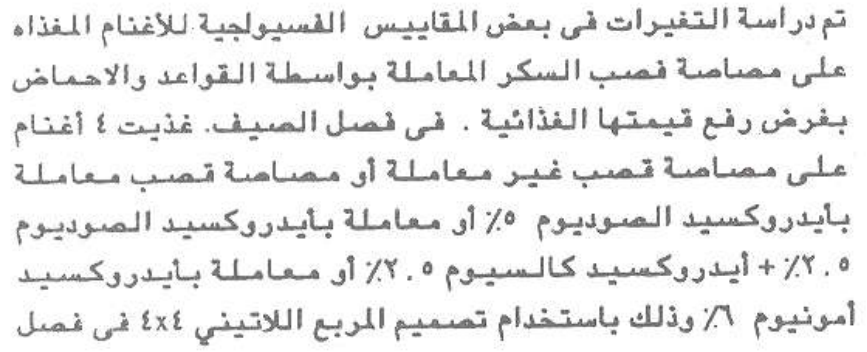

Egypt.J.Anim. Prod., 28, No.2 (1991) 


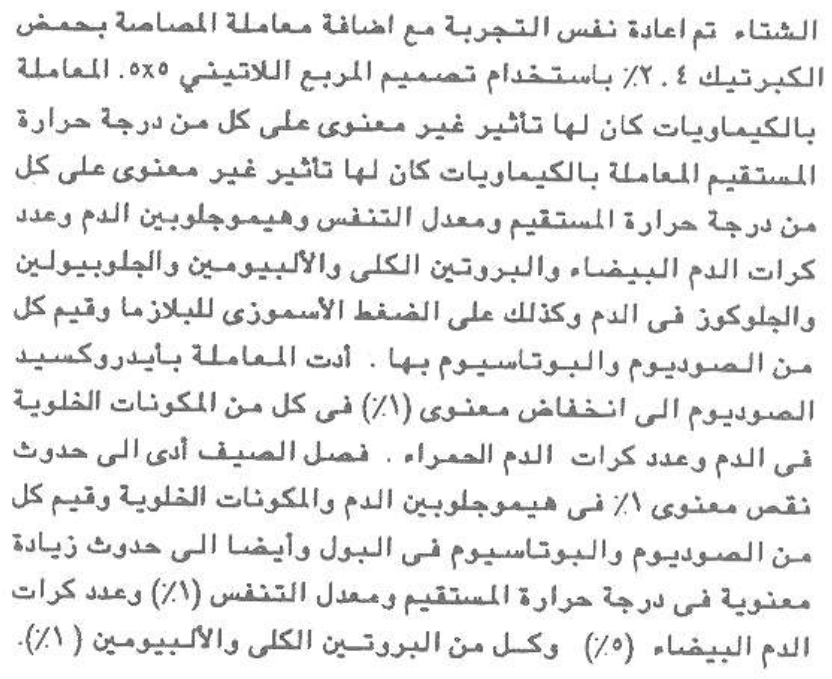

